\title{
Rêves amers et Conte cruel : les jeunes migrants de Maryse Condé
}

\author{
https://doi.org/10.29173/af29431
}

\section{(c) (i) $\ominus$}

\author{
Pooja Booluck-Miller \\ pooja.booluck@ubc.ca \\ Université de la Colombie-Britannique, Canada
}

Résumé. Cet article analyse la manière dont Maryse Condé dépeint les luttes auxquelles sont confrontés les enfants migrants dans ses romans Rêves amers et Conte cruel, qui mettent en scène des protagonistes enfants contraints d'assumer des responsabilités d'adultes afin d'aider leur famille. En tant que porteparole de ces enfants, Condé tente d'utiliser leur voix pour sensibiliser les jeunes lecteurs à leurs difficultés. Nous comparerons également ces deux romans pour la jeunesse à ses romans autobiographiques afin d'établir un lien entre les deux œuvres.

Mots clés : migration; littérature jeunesse; littérature antillaise; restavek; exil

\begin{abstract}
This article examines how Maryse Condé portrays the struggles faced by migrant children in her novels Rêves amers and Conte Cruel. These two novels depict how child protagonists are forced to assume adult responsibilities in order to help their families. As a spokesperson for these children, Condé uses her voice as an author to render awareness about their difficulties to young readers. We also compare these two children's novels to her autobiographical ones in order to establish a connection between the two.
\end{abstract}

Keywords: migration; children's novels; Caribbean Literature; restavek, exile

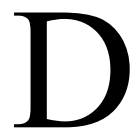
es sept textes de jeunesse écrits par Maryse Condé, quatre portent sur la migration, un déplacement humain compliqué, dur et fréquent qui touche beaucoup d'enfants autour du monde. Dans Rêves amers et Conte cruel, Condé nous présente deux personnages, Rose-Aimée, 13 ans et Tafa, 14 ans, qui sont déchus de leur enfance à cause des défis financiers et des pressions familiales. Rose-Aimée quitte sa terre natale en raison d'une sécheresse prolongée pour se trouver un emploi. Tafa est responsable de la famille de son frère, qui s'est exilé à Dubaï pour des raisons similaires à celles de 
Rose-Aimée. Cette étude traite du sujet de la migration juvénile dans ces deux textes et s'attache à montrer de quelle façon Condé dévoile à un jeune lectorat la chosification des enfants migrants. Nous noterons également l'utilisation de l'intertextualité pour faire ressortir l'aspect socioculturel des Antilles, cause fondamentale de la migration juvénile, et la façon dont elle initie le lecteur non antillais à un univers qui lui est inconnu. Sa voix d'auteure et quelques-unes de ses œuvres permettront de découvrir comment elle emploie la littérature jeunesse non comme un outil pédagogique ou didactique, mais plutôt pour faire réagir le jeune lecteur face aux questionnements liés à la migration et à l'identité.

\section{LES JEUNES MIGRANTS DE CONDÉ : ENFANTS OU ADULTES?}

Dans un dossier intitulé, La Caraïbe et le livre de jeunesse, Condé explique, « je ne suis pas un écrivain à message. J'écris d'abord pour moi, pour m'aider à comprendre et supporter la vie. En racontant des histoires que j'espère signifiantes, je souhaite aussi aider les autres, ceux de mon peuple en particulier, à comprendre et à la supporter à leur tour » (38). Dans ses œuvres pour la jeunesse, Condé opte pour une écriture particulière, une écriture qui dévoile la dure réalité à laquelle les jeunes Antillais font face, mais qui leur est aussi familière. Rêves amers évoque l'enfance perdue d'une petite Haïtienne, Rose-Aimée. Cette histoire raconte le parcours d'une restavek, qui habite et travaille chez une mulâtresse, afin de pouvoir subvenir aux besoins de ses parents. Françoise Lionnet met en lumière ce que signifie le mot restavek: « [an] estimated 300,000-400,000 children between the ages of 4 and 18 [are] sent by their desperately poor rural parents to live in urban households where they are treated as modern slaves and typically live as outcasts in the one-room homes of the barely better-off families they serve » (231). Malgré son chagrin d'être séparée de sa famille, Rose-Aimée avait des rêves comme aller à l'école, un droit humain qu'on considère comme un privilège en Haïti. Malheureusement, « mal nourrie, brutalisée, rudoyée », elle s'aperçoit très vite que madame Zéphyr, une « mulâtre », ne lui permettra jamais de s'instruire (Condé 28). Après cette expérience déplorable, Rose-Aimée cherche un emploi dans une chaîne de restauration rapide, où « elle allait être engagée précisément parce qu'elle était sans défense et qu'on pouvait tout exiger d'elle » (51). Il faut porter attention au comportement du patron, monsieur Modestin, lors de l'embauche. Il « éclata de rire » face au trouble de Rose-Aimée de ne pas savoir ce qu'il voulait dire par « papiers d'identité » et « paria » et qu'elle ne savait ni lire ni écrire (51). Rose-Aimée se rend compte que tous ces éléments négatifs qu'elle soulevait étaient en fait des facteurs que monsieur Modestin cherchait chez ses employés : enfants, illettrés, prêts à tout faire pour un peu d'argent. L'équipe dans laquelle se trouve Rose-Aimée comprend trois filles et un garçon, qui commencent à travailler à quatre heures du matin, bien avant celle chargée de la cuisine. On se demande si le patron les fait venir tôt afin que personne ne puisse se douter de ses actes illégaux. Certains patrons profitent de l'analphabétisme et de la vulnérabilité des enfants migrants : loin de leurs parents et de leur foyer, ces enfants sont subjugués par leurs employeurs jusqu'à ce que la malveillance de ces derniers devienne insupportable. Le travail des enfants est encore présent dans de nombreux pays du monde. Alors qu'ils se déplacent dans l'espoir de recevoir une éducation et de nourrir leur famille, ils sont malheureusement soumis à un sousproduit du colonialisme : « en 2001, le nombre de restaveks haïtiens était estimé à 300000 , dont $75 \%$ de femmes » (Enz 555, notre traduction). Ces enfants sont encore traités comme des esclaves ou des travailleurs sous contrat au profit d'employeurs qui négligent leurs droits légaux. D'où la nécessité d'une écriture de jeunesse inhabituelle visant à la fois la mouvance internationale de la migration et l'exploitation systémique des enfants dans le monde. Ce sujet pourrait a priori concerner les jeunes du monde entier dans la mesure où la condition scandaleuse de Rose-Aimée est celle de toute une génération et de toute une époque désemparée par l'insanité héritée du colonialisme. Et pourtant, ce n'est ni le thème des fictions populaires ni la matière habituelle de l'écriture pour les jeunes. Condé a donc beau récuser dans ses entrevues le rôle d'écrivaine à message, sa communication implicite est claire. Caractérisant les 
deux patrons comme des mulâtres qui abusent et profitent du dénuement des enfants noirs, elle nous amène à l'esclavage dit moderne. Pour Élise Saincotille, « certains Haïtiens, souvent issus des anciennes familles de maîtres esclavagistes, constituent à présent une classe aisée qui a conservé des habitudes de domination, et qui méprise les paysans » (para. 35). On voit plusieurs autres références au colonialisme, dont l'emploi du frère de Rose-Aimée qui disparaît après être parti travailler dans les cannes à sucre et le témoignage d'un homme revenant de ce même travail : " on [leur] a fait descendre dans un centre clôturé de barbelés, avec des soldats et des chiens [et que] certains se sont révoltés et on leur a dit qu'ils avaient été vendus » (Condé 75). Les substantifs « clôturé de barbelés » et « chiens » renvoient à l'image des cachots où l'on enfermait les esclaves qui n'osaient pas maronner, ayant peur d'être tués par des molosses. De ce fait, on comprend que le frère de Rose-Aimée ne montre aucun signe de vie à cause du fait que lui et ses confrères ont été vendus comme leurs ancêtres. Ces références subtiles de l'auteure renvoient à son propos : transmettre aux jeunes des connaissances au sujet de l'Histoire coloniale. Pour Kodjo Attikpoé, « la littérature pour enfants et pour la jeunesse ne demeure pas insensible aux problèmes qui se posent dans l'Afrique [et les Antilles] postcoloniales » (28). Il convient pour cela de comprendre pourquoi elle dit qu' « aujourd'hui plus que jamais il est bon d'intéresser les jeunes caribéens à l'histoire coloniale. Les migrations, la globalisation non élucidée, ajoutent à la complexité du monde » (Lebon 38). Condé tisse des événements importants liés à l'Histoire des Antillais tout en démontrant comment ils sont toujours pertinents dans la vie actuelle de Rose-Aimée. La condition postcoloniale et l'invisibilité qui se lie aux sans-papiers rendent le parcours migratoire des enfants migrants particulièrement difficile. Conte cruel, le deuxième roman que nous proposons d'analyser traite d'un problème migratoire à l'intérieur du continent africain. Ainsi, nous pouvons constater dans la littérature de jeunesse de Condé un prolongement de son rapport déjà long, complexe et problématique avec l'Afrique. Non seulement a-t-elle passé une partie importante de sa vie en Afrique et fait du continent la scène des romans qui ont assuré sa célébrité, mais elle s'exerce aussi dans un courant panafricaniste qu'elle a beau ridiculiser chez ses contemporains. Ainsi dans la vision d'une écriture mondialisée et politiquement consciente adressée aux jeunes, elle a envisagé la circulation du savoir sur la condition des enfants migrants à travers l'étendue globale de la diaspora africaine. De la même façon que les mésaventures de Rose-Aimée révèlent aux jeunes nord-américains, français et antillais un problème concret et actuel, les mésaventures de Conte cruel illuminent une dimension éminemment africaine de ce que Condé a toujours conçu comme un drame historique universel : l'enfant qui n'est pas chez lui dans le monde.

Dans Conte cruel, Tafa et Dafy, jeunes Guinéens, sont forcés de prendre des mesures drastiques face à une sécheresse qui entrave leur travail comme bergers nomades ${ }^{1}$. Incapables de se déplacer à cause d'un « nouveau pouvoir [qui] venait de forcer [les bergers] à se sédentariser dans la région », ils ne trouvent pas de quoi nourrir leurs bêtes (13). En utilisant le terme « le nouveau pouvoir », Condé fait une référence tacite aux grands changements intervenus dans des pratiques culturelles africaines à cause de la colonisation européenne, de politiques souvent mal conçues des régimes africains post-Indépendance et des affres de la mondialisation en général. En raison également d'une sécheresse prolongée (allusion possible au changement climatique), les villageois les plus riches s'exilent en ville tandis que les moins fortunés acceptent des travaux dont personne d'autre ne veut. D'autres s'exilent pour échapper à la famine. Dans cette optique, les habitants locaux comme Dafy, marié et père de quatre enfants, s'exilent à

\footnotetext{
${ }^{1}$ Nous supposons que le récit se passe en Guinée puisque la ville citée est « Tumbo », qui était nommée à l'époque, l'île de Tumbo, une partie de la ville de Conakry. Condé a vécu à Conakry pour une longue période de sa vie, ce qui explique probablement le choix de ce nom dans Conte cruel.
}

Alternative francophone

https://journals.library.ualberta.ca/af/index.php/af 
Dubaï, laissant la responsabilité de sa famille à son petit frère. Entre temps, Tafa vend leur vache préférée, Marafoudian, pour acheter des produits de première nécessité. L'animal sacré le remercie pour ses soins en lui offrant un don qui le sortira de la pauvreté : «à chaque fois que tu éprouveras du chagrin, un vrai chagrin, et que tu pleureras de vraies larmes, celles-ci seront métamorphosées en or et pierres précieuses » (9). Tafa vend Marafoudian parce qu'il ne reçoit aucun sou de Dafy alors que celui-ci s'est exilé dans le but de nourrir sa famille. L'aspect fantastique du don de l'animal suggère que le pays natal présente parfois des possibilités de ressources financières que certains migrants vont chercher ailleurs. C'est précisément ce que Tafa fait de la première quantité d'or qu'il reçoit de la vache : il se met en route pour Tumbo, la ville où se trouve un bijoutier. Là-bas, il se fait victime d'une société affamée d'argent. Le bijoutier qui essaie d'acheter la perle est fasciné par sa qualité, mais il rabaisse le coût en disant qu'il y a des taches brunes qu'il faut purifier (49). En revanche, sachant « qu'il assistait à une comédie réglée d'avance ", Tafa ment en disant qu'il a besoin de l'argent pour que son frère, atteint de tuberculose, soit guéri (33). Malgré son jeune âge, il comprend précisément la malice des adultes qui profitent des vulnérables. En fin de compte, Tafa obtient une somme convenable pour sa vente. Pour Condé, « les personnages d'enfants ne se contentent pas d'être les spectateurs des événements ou des tragédies vécus, ils en deviennent aussi les principaux protagonistes et les témoins actifs, n'hésitant pas à s'engager pour dévier le cours [des événements] » (Bonnet 2). Dans le contexte de la migration comme tragédie et devant les exploiteurs qui cherchent à profiter de la vie des jeunes victimes, nos deux protagonistes formulent un geste de révolte laissant un message clair selon lequel ils comprennent parfaitement bien les machinations du monde adulte. C'est pour cela que l'auteure se focalise sur l'intelligence et la maturité des deux personnages, enfants déplacés qui n'en luttent pas moins contre l'injustice.

De son côté, après avoir reçu des coups de ses deux patrons, Rose-Aimée décide de les quitter. La voix narrative offre ce commentaire : « la vie, c'est comme une bête qu'il faut dompter. Il faut bander ses muscles comme un pêcheur mettant à l'eau une pirogue rétive. À tout moment, la lame risque de la submerger, de l'emporter. Néanmoins, il tient bon » (61). Ici, Condé utilise l'allégorie d'un pêcheur qui confronte courageusement une bête pour démontrer la force et le courage qu'il faut avoir pour faire face aux difficultés. Dans son roman autobiographique, La vie sans fards, elle nous fait part de ses défis dans toutes les parties de sa vie : amour, carrière, famille, nationalité. Malgré son expulsion du Ghana, ses échecs amoureux, son parcours maternel, elle ne se laisse pas emporter. Dans Conte cruel, elle se permet un point ironique qui fera penser ses lectrices, « [...] les filles, qui s'amourachent du premier venu » (13). Ici, elle représente ces réflexions critiques à travers les actions du beau-père de Dafy qui espère marier sa fille « au prétendant le plus offrant » (ibid). Le beau-père n'est pas heureux d'apprendre que sa fille, Kira a épousé Dafy, qui selon lui est un étranger à cause de son titre de berger nomade. Malheureusement, la sécheresse obligeant Dafy à partir pour l'étranger, Kira meurt du chagrin causé par l'abandon de son époux. L'autobiographie de Condé nous apprend que celle-ci a beaucoup souffert à cause de ses expériences amoureuses : alors que deux hommes l'ont laissé pour se marier avec quelqu'un d'autre, un troisième la quitte pour poursuivre ses ambitions politiques. Les critiques universitaires l'ont également qualifiée de « nomade » en référence à son errance où elle brise les frontières littéraires et politiques (Bonnet 88). Ses voyages lui permettent également de faire vivre les émotions liées à la migration, comme la nostalgie et le deuil. Pour revenir à ce qu'elle dit dans une entrevue, Condé a beau avoir ses opinions au sujet de sa « vie scélérate », ses œuvres encouragent les jeunes nomades à mieux se préparer pour les mystères de la migration, quels que soient leur emplacement et leur condition.

Rêves amers et Conte cruel démontrent que les enfants du monde ne bénéficient pas tous des mêmes avantages. Alors que l'un peut se procurer un livre pour se familiariser avec les langues du monde, l'autre doit se déplacer pour épargner à sa famille la famine. Dans des textes destinés aux enfants de l'âge de huit ans et plus, Condé aborde des problèmes fondamentaux de la vie humaine. Ses protagonistes sont sujets à 
une infériorité « non pas à cause de leurs qualités innées, mais plutôt à cause des circonstances sociales paralysantes qui ont un impact sur leur vie [...] » (Enz 559, notre traduction). Par exemple, Rose-Aimée et ses sœurs doivent sacrifier leur enfance pour que leurs aînés ne meurent pas de faim. La sécheresse étant la source de la perte de travail de leurs parents, les filles se trouvent désormais dans des maisons à faire le ménage des inconnus. Quand Rose-Aimée souffrait aux mains de madame Zéphyr, ses sœurs «ne semblaient pas mécontentes. Elles étaient proprement vêtues et elles remettaient à leurs parents des gourdes qui permettaient de manger de la viande pendant quelques jours » (Conte cruel 10). Cependant, on ne sait guère comment leurs employeurs les traitent — il se peut qu'elles fassent semblant d'être heureuses sachant que leur famille compte sur elles pour survivre. Cette scène fait également allusion aux migrants qui, inconsciemment, font preuve de duplicité : ils présentent le pays d'accueil d'une manière indûment idéaliste et optimiste tout en passant sous silence les enjeux liés à la migration. Cela sert à préserver l'image de l'Eldorado de l'ailleurs, mais évite toute remise en question de leur bravoure et résilience (voir l'homme de Barbès dans Le ventre de l'Atlantique de Fatou Diome). Nous nous demandons si la situation de Rose-Aimée et de ses sœurs reflète cette observation, car pour elles, se lamenter sur le sort de la migration n'est pas une option.

Même après avoir subi la cruauté de ses deux patrons, Rose-Aimée n'ose pas rentrer, car elle sait que cela aggraverait la situation de ses parents (28). On assiste à la transformation d'une jeune fille qui au début ne faisait que pleurer à cause de son sort, mais qui finit par embarquer dans un bateau pour aller travailler en Amérique. Il est peut-être impensable qu'un enfant puisse entreprendre de tels plans, mais l'auteure nous apprend qu'à cause de la corruption et de l'exploitation de ces jeunes Antillais, ces derniers font preuve d'initiative et d'ardeur. Pareillement, dans Conte cruel, dès qu'il reçoit le don de Marafoudian, Tafa met en place une stratégie pour économiser de l'argent pour la famille de son frère : pour se protéger des voleurs, il demande à son camarade de le déposer en ville au lieu de se fier au long voyage par autobus. À un si jeune âge, il sait comment se protéger contre des délinquants grâce à l'attention méticuleuse qu'il porte à son alentour. Après avoir été battu par un groupe de jeunes voleurs qui vole aussi son sac à dos, il se sent reconnaissant d'avoir l'idée de " placer entre sa peau et ses sous-vêtements, comme il l'avait fait de sa pépite, les billets qu'il avait reçus chez le bijoutier » (39). Antje Ziethen cite Daniel Delbrassine qui note les étapes qu'entreprend le personnage type de la littérature jeunesse : « [le sujet] traverse un parcours initiatique qui se compose de quatre étapes : séparation, réclusion, métamorphose, révélation » (90). Condé emploie la même trajectoire dans les deux œuvres à l'étude : Rose-Aimée et Tafa débutent tout seuls dans leur cheminement vers l'inconnu, ce qui suscitera leur métamorphose en des êtres forts et courageux.

Néanmoins, étant donné la maturité et l'astuce démontrées par les deux protagonistes, la question se pose de savoir si la migration ne provoque pas un déficit d'enfance étant donné qu'ils doivent passer directement à l'âge adulte. Rose-Aimée et Tafa agissent-ils comme le ferait un enfant dans des situations qui incitent une réaction enfantine? Après avoir accompli la tâche qui lui a été confiée par madame Zéphyr, Rose-Aimée rencontre un groupe d'enfants qui jouent au ballon. Il convient de voir ses sentiments à l'intention de ces jeunes : « ah, que c'était bon de crier, de courir dans tous les sens, de partager l'excitation de garçons et de filles de son âge ! Il [lui] semblait que depuis qu'elle avait quitté les siens, elle n'avait pas ri, chanté, communiqué avec autrui. » (32). Grâce à cette période de relâche, elle retrouve son enfance, ce qui lui permet d'oublier brièvement ses enjeux quotidiens. On voit une scène comparable dans Conte Cruel quand Tafa envie l'innocence et l'insouciance des enfants qui jouent au cerf-volant : " comme il aurait voulu se mêler à ces garçons et bondir, lui aussi, la tête levée, un bout de

Alternative francophone

https://journals.library.ualberta.ca/af/index.php/af 
rêve, attaché au poignet ! [...] Comme il aurait aimé être lui-même un cerf-volant, libre et souverain, qui semble n'avoir aucune attache avec la terre !» (29). L'utilisation du cerf-volant comme métaphore implique le désir d'une liberté malheureusement refusée à Tafa. Il reconnaît qu'il ne peut guère profiter librement de son temps comme le font les enfants qu'il observe. Condé dédie Conte Cruel à l'auteur de The Kite Runner qui met en scène le rôle du cerf-volant pour les enfants afghans : « je remercie Khaled Hosseini, auteur du beau roman The Kite-Runner, qui m'a donné l'idée de ce conte cruel » (4). Le roman de Hosseini montre que le vol du cerf-volant est un symbole de liberté avant la prise de contrôle de l'Afghanistan par les talibans. Tout comme Tafa, Hassan et Amir sont pris dans des conflits créés par des adultes, tels que les régimes oppressifs et la dynamique familiale. De son côté, au lieu de profiter de sa liberté temporaire, Tafa refuse de jouer avec les enfants qu'il croit plus privilégiés que lui. Se croyant inférieur, il dénonce leur amitié à cause de son air de "paysan ». Selon lui, sa présence les offusquerait (39). Étant donné qu'il n'est pas de ce quartier, il ne se fie qu'aux apparences. L'étiquette attachée à l'étranger renvoie à la subordination et au doute, des sentiments provoqués par l'idéalisation d'autrui au détriment d'une estime de soi et de ses valeurs personnelles. Tafa est si aveuglé par son dénuement et par ses responsabilités comme chef de famille qu'il refuse l'illusion de son affranchissement. Bref, cette scène démontre la complexité de la réalité de ces enfants qui hésitent à agir conformément à leur âge en raison des différentes constructions systémiques liées dans ce cas aux problèmes de la migration. Cette scène est dans les deux romans plutôt positive comparée à d'autres scènes, mais ce qui suit est plus troublant : alors que Rose-Aimée s'aperçoit du vol de l'argent de madame Zéphyr après son bain avec ses nouveaux amis, Tafa se fait battre par un enfant voleur qui l'observait depuis son arrivée dans le quartier. L'auteure met l'accent sur le milieu cauchemardesque dans lequel se trouvent ces personnages, milieu où les enfants se battent pour se procurer quelques sous. En introduisant ce point culminant, voire perturbant, dans l'histoire, Condé esquisse une représentation claire de la marginalité des enfants migrants. En outre, malgré la perte de leur innocence et l'exploitation dont ils souffrent, Rose-Aimée et Tafa désirent librement s'épanouir; désir que les conditions concrètes de la migration n'ont pas, du moins chez Condé, complètement effacé.

\section{LA LITTÉRATURE NOIRE JEUNESSE NOIRE SELON CONDÉ}

Attikpoé met l'accent sur l'analyse culturelle des textes pour la jeunesse : « l'un des objectifs majeurs qui sous-entendent l'émergence d'une littérature pour la jeunesse autonome est d'ordre culturel. Elle remplit à l'origine une fonction culturelle, identitaire, portée par un discours sur soi » (27). Certains auteurs caribéens tels que Maryse Condé, Gisèle Pineau et Joseph Zobel passent de la littérature pour adultes à celle pour la jeunesse afin d'entreprendre la responsabilité de faire connaître à leur jeune lectorat le monde insulaire. Bien qu'il y ait très peu de textes pour jeunesse rédigés par des auteurs originaires de communautés marginalisées, ceux qui en écrivent s'assurent d'aborder des thèmes pertinents à l'Histoire et à l'identité antillaises. C'est pour cela qu'en adoptant une telle trajectoire, Condé fait vivre les cultures et les traditions inédites grâce à une écriture accessible et non aliénante pour un lectorat non antillais.

Renato Ulloa Aguilar note que « [...] les thèmes du surnaturel, du passé, de la vie rurale, des mœurs et des traditions sont présents dans tout l'espace géographique constitué par l'archipel des Antilles » (169). Dans Rêves amers, Condé exploite le sujet du surnaturel en évoquant des loas, qui sont des « esprits intermédiaires entre Dieu et les hommes » (14). Rose-Aimée les interpelle à plusieurs moments pour leur demander de l'aide ou pour les remercier. Par exemple, après avoir reçu du travail chez monsieur Modestin, elle remercie papa Legba et mère Ersulie. Saincotille explique que « Papa Legba et mère Ersulie sont deux esprits du vaudou. Papa Legba est toujours le premier et le dernier invoqué lors des cérémonies $[\ldots]$ quant à mère Ersulie, elle symbolise la beauté, la séduction. Elle est un des esprits les plus populaires du vaudou » (para. 15). En effet, on voit la description de mère Ersulie comme « si belle 
avec ses robes colorées et ses colliers de fleurs » et Papa Legba qu'elle évoque avant de commencer son chemin vers Port-au-Prince (Rêves amers 14). De même, quand monsieur Modestin lui donne un coup de pied, elle ressent « l'esprit de ses ancêtres africains, qui avaient conquis leur liberté en battant les puissantes armées envoyées par Bonaparte, la [posséder] à nouveau » (58). Condé partage avec ses lecteurs des histoires (coloniales) et des traditions haïtiennes qui attribuent une si grande importance aux esprits et aux ancêtres qu'on les inclut dans le quotidien, que ce soit pour des remerciements ou pour une rébellion.

De la même façon, elle utilise l'intertextualité pour se rapprocher de l'imaginaire de l'enfant. En étant exposé à des contes antillais, le lecteur s'ouvre à un monde soit étranger soit familier tout en permettant au non-Antillais d'élargir sa sensibilité culturelle. Contrairement à la migration qui est un phénomène global connu dans le monde entier, les pratiques culturelles doivent être clairement introduites afin de faire comprendre aux lecteurs et lectrices de diverses cultures le rapport intime entre les réalités culturelles antillaises et le problème de la migration qui n'a jamais été complètement absent de l'expérience historique des Antillais. Ziethen cite Clare Bradford qui observe que « les romans dotés d'une dimension multiculturelle (et écrits par des auteurs appartenant à un groupe ethnique/culturel particulier) » cherchent à offrir aux enfants autochtones des expériences de subjectivité narrative tout en permettant aux enfants non autochtones de s'engager dans la différence culturelle (92). Prenons le conte «Malice et Bouki », nom que Condé cite dans Rêves amers. Si l'enfant haïtien risque de comprendre les « malheurs » associés à cette histoire, l'enfant hors de l'espace caribéen ne saurait pas de quoi il s'agit. Pareillement, un conte africain représenté dans Conte cruel fait réfléchir au désir chez les femmes de ne concevoir que des garçons : une femme qui ne pouvait avoir d'enfants et qui fut méprisée par ses coépouses demanda à un génie de lui procurer un fils. Ce dernier, voulant lui épargner la maternité, accorda son vœu après plusieurs implorations de la femme. À peine a-t-elle accouché, le fils tua le père et ensuite la mère (21-23). Remarquons que Condé n'impose pas au lecteur les thèmes de la maternité ou du féminisme (la mère voulant un fils et non une fille). Son seul but est de le rendre spectateur d'un monde africain où la polygamie est prégnante et la conception des enfants du sexe masculin est valorisée chez certaines familles. L'intertextualité chez Condé, sert d'outil d'instruction. Attikpoé cite Klaus Doderer pour révéler les fins moralisatrices de certains auteurs de la littérature jeunesse :

Du moment que la littérature enfantine et pour la jeunesse est perçue comme un instrument — quelle que soit la forme - au service de l'information, de l'invitation à la raison, de l'éducation nationale [...], elle perd cette substance immanente à laquelle la littérature doit sa raison d'être et par laquelle elle peut produire son effet : la sensibilité. L'intention pédagogique dénature la littérature (26).

Un texte de jeunesse didactique risque de perdre l'intérêt de l'enfant qui, au lieu d'apprécier sa lecture, s'ennuie face à un livre moralisateur. En remplissant son œuvre de morales et de faits historiques étrangers à l'expérience de l'enfant, l'auteur risque de perdre son lectorat. C'est pour cela qu'il convient de laisser les jeunes déchiffrer par eux-mêmes le message transmis.

Maryse Condé a-t-elle des buts pédagogiques en écrivant pour les jeunes? Rêves amers et Conte cruel montrent que même si elle ne se voit pas comme écrivain à message, elle l'est, mais avec une approche différente. Comme elle le dit dans une entrevue, elle écrit de prime abord pour répondre à la demande de ses petits-enfants qui se sont plaints de son écriture dédiée seulement à leurs aînés (Lebon 38). Il est clair que la transmission est celle d'une grand-mère à ses petits-enfants (en raison du ton utilisé et de l'accessibilité de la langue). Sa transmission de contes ne sert pas seulement à instruire le jeune lecteur au

Alternative francophone

https://journals.library.ualberta.ca/af/index.php/af 
sujet de certaines vérités, elle l'invite à réfléchir sans que le message soit délivré de façon explicite. Par exemple, dans Conte cruel, Condé dévoile la dure réalité des sans-papiers dans la ville de Tumbo. Les habitants du pays voisin, issus du même destin que ceux de Tumbo, enjambent la frontière croyant qu'ils se procureront un travail. Le gouvernement a mis des militaires sur place pour contrôler ce flux migratoire illégal, ce qui provoque de la confusion chez Tafa : « que viendraient [les migrants illégaux] de toute façon chercher dans ce village qui n'avait à offrir que sa misère? À part l'arrivée des militaires, rien de nouveau n'était à signaler depuis des années » (16). Ses questions soulignent le désespoir des sans-papiers qui commencent leur voyage sans savoir que le pays d'accueil souffre d'un manque de ressources et de nourriture. Le gouvernement dépense son argent sur le contrôle des frontières au détriment de ses habitants. Les prix des produits alimentaires sont exorbitants rendant les citoyens sans moyen pendant des jours. Cependant, la nouvelle génération ne partage pas les mêmes réserves que Tafa à l'égard des étrangers. Avec le peu d'argent qu'il a, Tafa achète deux oranges pour ses neveux qui au lieu de le remercier lui reprochent le fait qu'ils ne pourront pas les partager avec leurs camarades. Cette réponse le fait réfléchir à l'époque où Dafy et lui étaient plus jeunes. Avant la construction des frontières, les gens se déplaçaient comme ils voulaient. Les bergers de la famille de Tafa profitaient d'une vie aisée grâce au déplacement requis pour un tel travail. Malheureusement, à cause de l'exigence des colonisateurs, « [...] les nomades avaient été forcés de se fixer d'un côté ou de l'autre de la frontière sous peine d'être déclarés apatrides et d'être pourchassés » (18). Cette ségrégation renforce le sentiment de conflit chez Tafa, qui a été privé de ses droits humains par des étrangers. Cela explique pourquoi il reste hébété devant ses neveux qui promeuvent le partage avec leurs camarades de classe, quel que soit leur milieu : « Le temps d'aujourd'hui n'était pas celui que Tafa avait connu. Pour [son neveu] Manthia, les “autres" n'étaient plus des ennemis. Ils étaient des camarades » (19). Le dénuement rapide de ce pays est la raison pour laquelle les écoliers agissent ainsi. Contrairement à l'époque de Tafa, la nouvelle génération reconnaît que la fraternité et la solidarité sont essentielles à la survie des habitants et non un conflit que les autorités ont manifesté. Cette scène est précisément le style de Condé. C'est au lecteur de tirer sa propre leçon basée sur l'information donnée. Elle nous révèle l'effet des frontières sur les habitants ainsi qu'une pensée moderne et humanitaire à cet égard.

Nous avons vu l'effort de Condé pour rapprocher le lecteur du monde culturel des Antilles, grâce à l'intertextualité. Elle cherche également à évoquer la nature puisque celle-ci occupe non seulement un rôle important dans les mœurs antillaises, mais elle est souvent le moyen utilisé pour le déplacement (l'air, l'océan, la terre). N'ayant pas d'autres options en Haïti pour nourrir sa famille, Rose-Aimée rejoint des clandestins pour se rendre en Amérique. Malheureusement, les garde-côtes les repèrent et ils sont forcés de se jeter à l' eau, même si certains parmi eux ne savent pas nager. Pour donner suite à cette scène, la narratrice dit « et la mer roula ces déshérités dans son suaire » (79). Rose-Aimée et ses compagnons sont des déshérités non seulement de la mer, mais aussi de deux terres, celle qu'ils ont fuie et celle qu'ils essayent d'atteindre. La mer les jette dans son suaire alors qu'ils se fiaient à elle pour trouver le salut. Cette scène fait allusion aux esclaves africains qui, désespérés, se sont jetés aussi à la mer. Pourtant, la façon dont Condé décrit la mer avant le naufrage prédit la mort des traversés : " la mer, cette bête indocile, avait changé d'humeur. Elle n'était plus douce et rampante, mais furieuse et agressive. Ses coups de reins faisaient valser le navire qui virevoltait, tournoyait... (76). Le contraste des adjectifs « douce et rampante » à « furieuse et agressive » souligne leurs illusions à l'égard de leur destination. Eux qui croyaient que la mer et l'ailleurs résoudraient leur problème, finissent par y rencontrer la mort. Toutefois, remarquons que la mer redevient calme après le saut des sans-papiers : « elle chanta de sa voix suave pour calmer les terreurs des enfants, de Rose-Aimée et de Lisa, et, les yeux fermés, ils glissèrent tous dans l'autre monde » (79). La personnification de la mer ici sert à évoquer l'image de confort et de sécurité, car seule la mort pourrait adoucir leur peine. On voit la mer comme élément du roman non 
seulement dans la littérature pour la jeunesse de Condé, mais aussi chez plusieurs auteurs africains et caribéens qui traitent de l'esclavage (Marie-Célie Agnant, Édouard Glissant, Léonora Miano) même si les intentions des esclaves qui se jetaient à la mer n'étaient pas exactement les mêmes. Condé ne se laisse pas influencer par la norme d'un « happy end» qu'on voit souvent dans la littérature pour la jeunesse. Au contraire, elle souligne la réalité des migrants qui meurent quotidiennement alors qu'ils ne voulaient qu'une vie meilleure. Le voyage de Dafy en est un exemple. Lui, qui pensait bénéficier d'une plénitude à Dubaï, revient au pays dans un état plus défavorisé qu'avant son départ : « Dafy avait beaucoup maigri [...] On sentait bien qu'avec son voyage, il n'avait pas abordé les rivages de la fortune et du bonheur » (45). Cela met en évidence la triste ironie chez les migrants qui abandonnent leur famille croyant que leur futur sera plus prometteur. Dans le cas de Dafy, une telle démarche met en péril l'enfance de son petit frère qui se retrouve soudainement chef de famille. Ce retour motivé par l'échec dans le pays d'accueil reste l'un des thèmes les plus abordés dans d'autres textes littéraires antillais et africains (Le ventre de l'Atlantique, Le baobab fou, L'exil selon Julia). En inscrivant cet échec dans la littérature jeunesse, Condé souhaite faire comprendre aux enfants que la migration ne promet pas toujours une traversée enrichissante. Ceux qui s'imaginent heureux en se déplaçant dans le monde n'ont pas été en règle générale ceux qui ont connu le poids d'une migration inévitable, sans l'assurance d'une vie meilleure. Que les jeunes qui lisent Rêves amers et Conte cruel s'imaginent heureux ou non, qu'ils sachent, nous suggère Condé, que cela n'a pas été l'expérience vécue de tous les enfants, que tous les enfants n'ont pas forcément la même perspective.

Le roman autobiographique, La vie sans fards, nous révèle les déplacements de Condé ainsi que les défis éprouvés dans chaque pays. Écrire la migration, pour elle, implique écrire ses expériences, à la fois douloureuses et fructueuses. Toutefois, on remarque une distinction dans la façon dont elle aborde ce sujet dans ses œuvres pour la jeunesse et ceux pour adultes tels que Desirada et Histoire de la femme cannibale. Même si les thèmes du racisme, de l'aliénation et de la collectivité sont présents dans les deux sphères, elle a une approche plus personnelle dans ses œuvres pour adultes. Par exemple, on retrouve quelques bribes de la vie de l'auteure à travers le récit de Marie-Noëlle (les deux sont nées en Guadeloupe, ont vécu en Europe et finissent par enseigner en Amérique). En revanche, bien qu'elle ait beaucoup écrit sur la violence et la clandestinité (Les belles ténébreuses, La belle créole, Le triste et fabuleux destin d'Ivan et Ivana), on s'aperçoit qu' elle a recours au merveilleux dans ses récits pour la jeunesse; un sujet que l'on voit aussi parfois dans ses romans pour adultes (Traversée de la mangrove, Moi Tituba, Sorcière... Noire de Salem, La vie scélérate), mais qu'elle incorpore à ses objectifs de représentation personnelle. Les romans de Condé écrits pour les adultes ont tendance à refléter son itinéraire personnel d'écrivaine migrante, itinéraire sans « happy end ». Dans les ouvrages écrits pour les jeunes tels que Rêves amers et Conte cruel, tout revient aux dures réalités des enfants jetés dans le maelstrom de la migration.

Dans Rêves amers, Condé consacre un chapitre, intitulé « les sans-abris », à la condition de certains jeunes Haïtiens qui se trouvent sans logis après leur quête d'un emploi convenable. Alors que certains d'entre eux fuient leur condition pitoyable de restavek, certains utilisent la cathédrale, près des détritus, pour mendier (39-40). En cherchant une place où dormir, Rose-Aimée se pose la question suivante : «pourquoi des peuples sont-ils riches, et d'autres si pauvres qu'ils doivent aller chercher hors de leur pays natal des moyens de subsister » (40). Une réflexion similaire est visible dans Conte cruel : «Tafa se demanda d'où sortaient les hommes qui prenaient ainsi leur petit déjeuner. N'avaient-ils pas de toit, d'épouse, de mère, de sœur qui puissent cuisiner pour eux ?» (27). Ni les protagonistes ni Condé

Alternative francophone

https://journals.library.ualberta.ca/af/index.php/af 
n'offrent de réponse à cette question, mais en la posant ainsi, l'auteure nous fait réfléchir à l'autre facette de la migration : celle où les gens, démunis des éléments essentiels pour survivre, doivent quitter leur pays. Ironiquement, quand Tafa, surpris de la réponse de son ami qui dit que ces hommes viennent chercher de l'argent, s'exclame que même les habitants locaux n'ont pas de travail. Les personnages clandestins des deux romans se sentent vaincus au point qu'ils commencent à errer, espérant qu'un pays les adoptera. Quant à Rose-Aimée, elle rencontre Lisa, une autre victime de la violence de sa patronne, qui lui propose de partir pour l'Amérique où elles pourront faire des économies. Malgré l'espoir de ces deux jeunes filles, elles se noient dans la mer. On s'interroge sur le message de Condé ici, car elle termine le roman en disant, « [...] la mort n'est pas une fin. Elle ouvre sur un au-delà où il n'est ni pauvres ni riches, ni ignorants, ni instruits, ni Noirs, ni mulâtres, ni Blancs... » (79). On se demande pourquoi elle considère la mort comme une fin positive tandis que pour plusieurs migrants, il s'agit d'un grand échec. Dans plusieurs œuvres de Condé (La migration des cours, La colonie du nouveau monde, La vie scélérate), « la mort est une sorte de "migration sans retour" (Angrey 655). Alors que certains personnages utilisent la mort comme source de révolte, certains la voient comme une quête de la paix (Angrey 656, 659). Dans Rêves amers, les personnages condéens se retrouveront enfin libres dans un monde où ils seront à l'abri de la discrimination. Les problèmes liés au statut social, à la couleur de la peau, à l'instruction et à l'argent ne les suivront pas dans « l'autre monde ». Il semble qu'en décrivant leur mort d'une manière poétique, Condé tente de s'approcher de l'effet d'un « happy end » tout en restant fidèle à la réalité de la migration. Quant à Conte cruel, même si Dafy retourne au pays à cause de la mort de Kira, on s'aperçoit à la fin de l'histoire que le don de Marafoudian disparaît. Il convient de voir comment Condé nous fait part de cette nouvelle qui changera la vie des deux frères. Elle commence la dernière page par la construction d'une nouvelle case pour la famille. Pour donner suite à cela, on peut espérer une fin heureuse grâce à la description de la nature et à la voix prometteuse de la narratrice,

[p]ar la fenêtre ouverte, il voyait un grand pan de ciel qui bleuissait lentement. Le soleil montait au fur et à mesure. Il sembla [à Tafa] qu'une nouvelle vie commençait. Moins dure, moins amère et moins ingrate que la précédente. Grâce à Marafoudian, ils auraient toujours de quoi manger à leur faim. Il n'y aurait personne entre Dafy et lui. Ensemble, ils élèveraient les enfants comme s'ils étaient à la fois leur père et leur mère. (47).

L'image du soleil qui se lève lentement fait allusion à la renaissance des deux frères qui ne se soucient plus de l'argent grâce au soutien financier de Marafoudian. Dans ce paragraphe, l'auteure utilise un ton vif, plein d'espoir, bref, ce à quoi l'on s'attend souvent après une réconciliation familiale longtemps désirée. En disant qu'il n'y aurait personne entre les deux frères, la voix narrative fait implicitement référence à Kira. La phrase suivante renforce le fait que Tafa, heureux d'avoir Dafy pour lui, est optimiste de pouvoir combler le vide de la mère des enfants. Dans cette lumière, Condé change subitement la fin du récit quand Tafa, se souvenant de Kira, pleure et « ne [remarque] pas tout de suite que [ses larmes] ne se [transforment] plus en or » (48). Ses sentiments à l'intention de sa belle-sœur sont à noter. Il la blâme pour l'exil de son frère et n'éprouve aucune pitié face à la décadence de celle-ci : « [...] il lui en voulait terriblement d'avoir mis au monde quatre enfants en rapide succession, obligeant ainsi le malheureux Dafy à s'exiler. Il n'éprouvait aucune pitié à la voir se faner chaque jour davantage, perdre sa jeunesse et ressembler de plus en plus à une vieille femme » (13). L'objectif de migrer afin de soutenir sa famille $\grave{a}$ l'avenir fait de Dafy un héros aux yeux de Tafa, contrairement à Kira qui ne reçoit aucune admiration malgré l'attention qu'elle prête à ses quatre enfants dans l'absence de son mari. Le don de Marafoudian exige que Tafa éprouve un vrai chagrin pour que ses larmes se transforment en perles. Nous avançons que les larmes versées par Tafa après la mort de Kira ne sont pas sincères, ce qui explique qu'elles ne se transforment pas en or. En terminant le récit ainsi, Condé nous entraîne vers la possibilité que Dafy étant 
de retour, Tafa n'ait plus besoin de soutien pour nourrir sa famille. On ne saurait que faire des hypothèses, mais les deux œuvres, malgré l'attente d'un « happy end », se terminent de façon négative, mais réaliste.

\section{CONCLUSION}

À la suite de la lecture de Rêves amers, on comprend pourquoi les éditions Bayard changèrent le titre original de l'œuvre de Condé en Haïti chérie (Saincotille para. 5). Rêves amers comme titre du roman caractérise mieux le parcours cauchemardesque d'un enfant-migrant. Il convient aussi de voir le caractère insolite des titres qu'elle choisit pour des récits de jeunesse : Rêves amers et Conte cruel contiennent une connotation négative et annoncent dès la couverture une histoire inquiétante, avec une fin malheureuse. En fait, le cheminement de Rose-Aimée et de Tafa se termine par une fin cruelle, où l'un trouve la mort et l'autre retrouvera le dénuement dont il a cru échapper. À travers le déplacement de Tafa à Tumbo pour faire monnayer ses perles et de Rose-Aimée pour subvenir aux besoins de ses parents, on constate que pareillement à un migrant qui s'éloigne de son domicile, les deux enfants sont victimes des pires cruautés, telles que les violences physiques et l'aliénation. Grâce au style particulier de Condé qui met à profit l'intertextualité, la culture antillaise et des messages peu moralisateurs, elle révèle aux jeunes les gageures de la réalité migrante sans une tentative de rendre moins amers les défis quotidiens. Bref, en lisant les aventures des jeunes héros de Condé, le lecteur prend conscience de la chosification et de l'exploitation de ces enfants noirs qui luttent sans soutien, malgré leur âge et leur invisibilité, contre les grandes problématiques du monde. 


\section{BIBLIOGRAPHIE}

Aguilar, Renato U. « Les caractéristiques du roman antillais à travers l'œuvre de Maryse Condé, Frédéric Régent et Joseph Zobel », Revista de Lenguas Modernas, no. 26, 2017, pp. 155-170.

Angrey, Francis U. « La mort ou "la migration sans retour"? Une lecture thanatico-psychologique des romans de Maryse Conde », Neohelicon, vol. 40, no. 2, 2013, pp. 655-664.

Attikpoe, Kodjo. «La littérature de jeunesse entre normes pédagogiques et littéraires : le cas des pays francophones d'Afrique », International Review of Education, vol. 53, no. 1, 2007, pp. 23-37.

Bonnet, Véronique. «La littérature de la Caraïbe pour la jeunesse : des histoires à part ou l'histoire à Part Entière ?», Amnis, vol. 16, no. 16, 2017, pp. 2-3.

-. «Quand les enfants et les adolescents font le politique : La littérature pour la jeunesse de Maryse Condé », Nouvelles Études Francophones, vol. 2, 2020, pp. 87-102.

Condé, Maryse. Rêves amers. Bayard Jeunesse, 2017.

—. Conte cruel. Mémoire d'encrier, 2014.

—. La vie sans fards, JC Lattès, 2012.

Enz, Molly Krueger. "The Haitian Flight for Freedom in Maryse Condé's Rêves amers and Marie-Célie Agnant's Alexis d'Haïti', Contemporary French and Francophone Studies, vol. 22, no. 5, 2018, pp. 553-561.

Lebon, Cécile et Maryse Condé. « Entretien avec Maryse Condé », La Caraïbe et le livre de jeunesse. Takam Tikou, 2002, cnlj.bnf.fr/fr/block-revue-numerique/entretien-avec-maryse-cond

Lionnet, Françoise. "Postcolonialism, Language, and the Visual: By Way of Haiti”, Journal of Postcolonial Writing: New Directions in Postcolonial Studies, vol. 44, no. 3, 2008, pp. 227-239.

Pfaff, Françoise. Nouveaux entretiens avec Maryse Condé. Écrivain et témoin de son temps. Karthala, 2016, pp. 157-158.

Saincotille, Élise. « C comme Condé », Abécédaire insolite des francophonies. Presses Universitaires de Bordeaux, 7 mars 2019, books.openedition.org/pub/3548

Ziethen, Antje. « La littérature pour la jeunesse ou l'art de "Danser dans les chaînes" : trois textes sur la diaspora haïtienne en Amérique du Nord », Francophonies d'Amérique, no. 33, 2012, pp. 79-94.. 